\title{
Card-type tool to support divergent thinking for embodying benefits of inconvenience
}

\author{
Yuichi Hasebe, Hiroshi Kawakami ${ }^{*}$, Toshihiro Hiraoka and Kosuke Naito \\ Department of Systems Science, Graduate School of Informatics, Kyoto University, Yoshida-Honmachi, Sakyo, \\ Kyoto 606-8501, Japan \\ E-mails: hasebe@sys.i.kyoto-u.ac.jp, kawakami@design.kyoto-u.ac.jp, hiraoka@sys.i.kyoto-u.ac.jp
}

\begin{abstract}
This paper proposes a card-type divergent thinking support tool to design systems that make use of FUrther BENEfit of a Kind of Inconvenience (fuben-eki). Even though the benefits of inconvenience are known as the essence of spiritually affluent lives, there are few artifacts for providing them. Our proposed tool borrows a leaf from TRIZ, which is the theory of inventive problem solving, based on the results of analyzing inconvenient tools and methods. Our experimental results show that principle cards, which are a part of the tool, increase the number of ideas when they are used according to guidelines that compel users to concentrate on creating ideas from each card.
\end{abstract}

Keywords: Human-machine system, fuben-eki, system design, divergent thinking, creativity support tool

\section{Introduction}

System designers aim to enrich our lives. A rich life is sometimes misunderstood as being full of conveniences. In this sense, full automation seems the ultimate goal of system design. However, this goal is not always the best for users or human-machine systems. On the contrary, the convenient auto-adaptation ability of machine may be harmful to human operator [1]. It spoils our human ability to acquire skills and maintain motivation. Nor should the pleasure of system usage be ignored. From this viewpoint, we focus on the richness obtained from the benefits of inconvenience that have been overlooked by pursuing only convenience. A systems theory of benefits of inconvenience is now being constructed to find the value of inconvenience [2]. However, few systems embody such benefits.

Although designs with the benefits of inconvenience can be devised by conventional brainstorming [3], their results depend on the experience and the knowledge of the participants. There is also no effective way to support divergent thinking for such designs. There-

\footnotetext{
${ }^{*}$ Corresponding author.

E-mail: kawakami@design.kyoto-u.ac.jp.
}

fore, this paper proposes a tool that supports divergent thinking to embody the benefits of inconvenience by referring to the results of analyzing its tools and methods.

\section{Introducing divergent thinking and fuben-eki}

\subsection{Outline of divergent thinking}

\subsubsection{Divergent thinking}

Human thought can be classified into divergent thinking and convergent thinking [4]. For creative problem solving, convergent thinking focuses on deriving a single, well-established answer. In the divergent thinking process, it is important for designers to create broad ideas even if the relevance to the problem is not clear [5]. To create good solutions, the processes that use divergent and convergent thinking should be divided.

\subsubsection{Brainstorming}

Brainstorming is a typical creativity technique using divergent thinking. To freely encourage divergent thinking, brainstorming has the following four principles [6]:

- Criticism is ruled out. 
- "Free-wheeling" is encouraged.

- Quantity is wanted.

- Combinations and improvements are sought.

Although brainstorming was originally a method for group interaction, creativity decreases when brainstorming is carried out in a group [7]. Accordingly, such revised methods as brainwriting were proposed [8].

\subsection{Brief introduction of fuben-eki}

\subsubsection{Convenience and inconvenience}

For discussing the benefits of inconvenience, this paper defines the following terms [9]:

$$
\begin{aligned}
\text { convenience } \equiv \text { saving labor to attain } \\
\text { a specific task } \\
\text { labor } \supset \text { time consuming } \\
\text { labor } \supset \text { special skills } \supset \text { mental load. }
\end{aligned}
$$

Based on the above definitions and since this paper regards inconvenience as the opposite of convenience, something inconvenient requires more labor for a particular purpose than something convenient.

\subsubsection{Harm of convenience}

Many convenient systems have made our lives richer. Even though convenient systems reduce labor and accomplish tasks efficiently, they also have harmful aspects.

An automatic system sometimes acts against users' will. Users cannot understand the behaviors of the system, when there is a communication gap between users and the system that leads to distrust and conflicts.

An automatic system sometimes prevents users from imagining its internal state. Some users cannot help checking the doors of their cars are locked when they operate remote controller. Although they can get such feedback as beep or blink of hazard-lamps, they cannot judge whether the feedback is of locking doors or of pressing the controller.

Excessive reliance on convenient systems decreases user abilities and chances for decision-making. This decreases their perceived competence and feelings of self-determination [10]. Convenient safety decreases the level of perceived risks and ironically encourages dangerous behavior [11].

\subsubsection{Benefits of inconvenience}

In terms of human-machine systems, convenience does not always enrich our lives. The benefits obtained from inconvenience are now being recognized [2]. We call such benefits that can be obtained not from convenience but from inconvenience FUrther BENEfit of a Kind of Inconvenience (fuben-eki), and build design methodology that positively embrace it [3]. Some case examples of fuben-eki are shown below:

A knife: Compared with an electric pencil sharpener, a knife requires more skill and effort for sharpening a pencil. However, the labor of using a knife increases user dexterity, and allows users to devise their own ways that lead novel shapes of pencil heads.

$$
\begin{gathered}
\text { physical effort } \rightarrow \text { increase in skill } \\
\text { difficulty } \rightarrow \text { devising ways }
\end{gathered}
$$

A manual transmission car: Compared with automatic transmissions, a manual transmission car requires more operation and skill. However, because of its difficulty, users can feel self-efficacy [12] when they drive such cars well. Moreover, it has functional transparency and users can make their own mental models of systems through the interaction.

$$
\begin{aligned}
\text { difficulty } & \rightarrow \text { self-efficacy } \\
\text { more operations } & \rightarrow \text { system comprehension }
\end{aligned}
$$

A gas cooker: Compared with an induction-heated stovetop, a gas cooker that uses fire is more dangerous and users spend more cognitive resources. However, its visible danger makes users more careful. On the other hand, because the danger of induction-heated stovetops is hard to notice, users may put something on the stovetop.

$$
\text { visible danger } \rightarrow \text { safe behavior }
$$

Prior research collected the benefits of inconvenience from many examples $[13,14]$ :

- fosters affirmative feelings,

- provides motivation to tasks,

- personalization [15],

- relaxes users.

We also get the following abstract suggestions for realizing these benefits:

- allows understanding of systems (mental visibility),

- allows exploration (enhancing awareness),

- allows creative contributions to tasks,

- allows limitless proficiency.

Of course, not every inconvenience has beneficial aspects. Fuben-eki is different from nostalgia that 


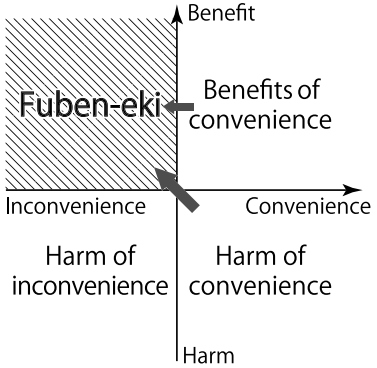

Fig. 1. Orientation of fuben-eki.

tends to return to traditional things or to design something appears retrospectively. Besides, inconvenience should be inevitable to get fuben-eki. In other words, inconvenience is not a compromise with fuben-eki. For example, time and effort to type in and to remember a password are only compromise with security. In this case, security is not fuben-eki. Typing in a password is not inevitable and can be replaced by other methods for security. However, if the process of typing a password induces such subjective benefits as feeling self-efficacy [12], the feeling is fuben-eki.

\subsubsection{Systems theory of fuben-eki}

The benefits and the harm of convenience and inconvenience are arranged as shown in Fig. 1. For system design, the harm of inconvenience is out of the question. The viewpoint of fuben-eki aims to remove the harm of convenience or to embody benefits of inconvenience. Systems theory of fuben-eki is a methodology that designs systems to exploit fuben-eki, even if that decreases the level of convenience. However, few systems apply this theory.

\section{Support tool for divergent thinking to design fuben-eki systems}

This section defines the terms of fuben-eki system design, and proposes a tool to support such a divergent thinking process.

\subsection{Fuben-eki system design}

\subsubsection{Definition}

Fuben-eki systems are defined as systems through which users obtain the benefits of inconvenience. In this paper, design processes convert existing systems into new fuben-eki ones.

The benefits of inconvenience introduced in Section 2.2.3 tend to decrease the convenience of systems. Conversely it is expected that making systems

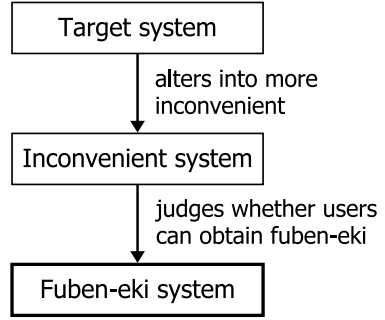

Fig. 2. Fuben-eki system design.

more inconvenient can give users fuben-eki [3]; i.e., one method to design fuben-eki systems is converting systems to require more labor to attain tasks.

Of course, it is insufficient to just alter the systems into inconvenient ones. We must judge whether the altered systems enable users to obtain the benefits of inconvenience by using them. Such subjective benefits as affirmative feelings or increased motivation to perform tasks, which are caused by the inconvenience process, are the goals of fuben-eki system design. The following is our definitions of fuben-eki system design:

- alters target systems to require more labor to attain tasks,

- judges whether users can obtain subjective benefits due to the extra labor.

In these definitions, target systems denote the systems converted into fuben-eki systems. Figure 2 shows the procedure of a fuben-eki system design.

\subsubsection{Fuben-eki system design and the Geneplore model}

The Geneplore model is a heuristic scheme of creative functioning, in which creativity takes place in two phases: generative and exploratory (Fig. 3) [16]. In the generative phase, a designer constructs mental representations called preinventive structures, which are used to devise creative ideas in the exploratory phase. Applying this model to fuben-eki system design, the phase to make the target system inconvenient can be seen as the generative phase, and the phase to explore fuben-eki is the exploratory phase.

\subsubsection{Divergent thinking process of fuben-eki system design}

When we use divergent thinking in the fuben-eki system design, we have to devise ways to make the target system inconvenient without concerning its feasibility or practicability. The course to alter the target system into an inconvenient one is not only counter to the actual historical development of the system. For example, for converting navigation systems to fuben- 


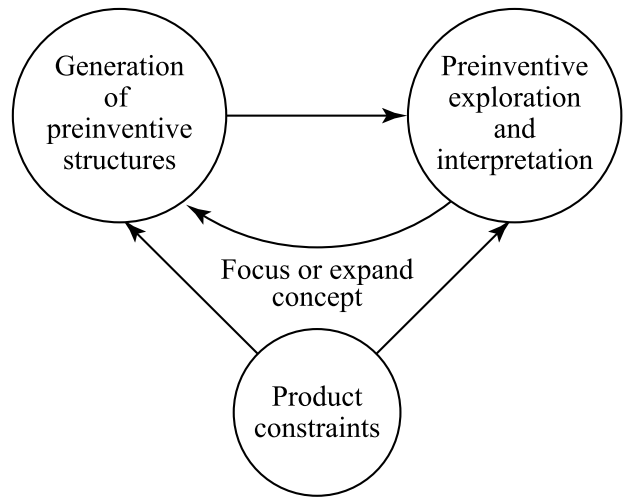

Fig. 3. Geneplore model [16].

eki systems, Kitagawa added a novel inconvenience: degradation of information [17]. In this navigation system, the trails through which users passed are getting disappeared, so users need to recall them when they use the system again and they remember landmarks more precisely.

To generate many ideas, we have to take account of as many parameters of inconvenience as possible. We also have to explore as many benefits as possible, which can be obtained from altered target systems. The benefits found in this phase can be applied when we polish the ideas or produce others.

\subsection{TRIZ and fuben-eki principle}

Although fuben-eki systems can be devised by brainstorming, the results depend on the experience and the knowledge of the participants. Focusing on this problem, Naito developed a support method for fubeneki system design inspired by TRIZ $[13,14]$. This section outlines TRIZ and Naito's method.

\subsubsection{Outline of TRIZ}

TRIZ $[18,19]$ is a theory of inventive problem solving based on the following findings:

- The top few percent of patents that generally overcome technical contradiction are inventive: the condition of improving one parameter negatively impacts another.

- The solutions of inventive patents can be categorized into 40 ideas (called principles).

As a tool exploit of these findings, TRIZ provides contradiction-matrices [18]. By choosing the improving and worsening features from the rows and columns, users can find inventive principles that solve technical contradictions at the intersection of the ma-

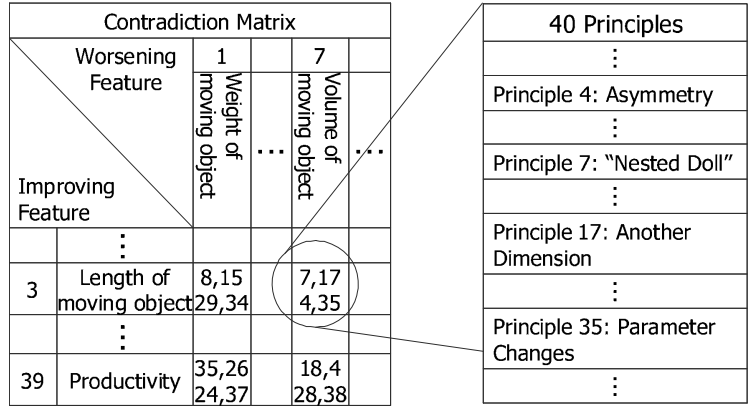

Fig. 4. Part of contradiction-matrix and TRIZ principles [13,14].

trix (Fig. 4). Users can learn the helpful principles if they model their own problem as a contradiction of two parameters.

\subsubsection{Fuben-eki principles and fuben-eki matrix}

In many cases trade-offs exist between subjective benefits, such as self-affirmation or a sense of security, and objective convenience. To support fuben-eki system design, Naito interpreted the above trade-off as the notion of technical contradiction in TRIZ and derived the fuben-eki matrix from analysis of the examples $[13,14]$. Table 1 shows the fuben-eki matrix that contains conveniences in the rows and the benefits of inconvenience in the columns. The IDs of the principles $(1,2, \ldots, 10)$ are placed at the intersections of the relevant convenience and the benefit. Table 2 shows the ten principles corresponding to each ID in the matrix as well as new principles (ID 11, 12) which were derived after the fuben-eki matrix was proposed. A web application to support fuben-eki system design was developed [20] that borrows a leaf from the fuben-eki matrix. However, the experimental result [20] shows that the application failed to increase the number of ideas, although it did improve the quality of ideas for people who utilized the application in depth.

\subsection{Fuben-eki cards}

For the fuben-eki system design, there is still no way to support the divergent thinking process. This paper aims to propose a case-based support tool by borrowing a leaf from the fuben-eki matrix and the fuben-eki principles.

The inventive principles of TRIZ can be used without a contradiction matrix. Users can chose and apply the principles at will. CREAX, which is TRIZ software, shows the principles randomly to support such divergent thinking processes as brainstorming. The fuben-eki principles can also be used without the 
Table 1

Fuben-eki matrix

\begin{tabular}{|c|c|c|c|c|c|c|}
\hline & $\begin{array}{l}\text { Enhancing } \\
\text { awareness }\end{array}$ & Devising ways & Improvement & $\begin{array}{l}\text { System } \\
\text { comprehension }\end{array}$ & $\begin{array}{l}\text { Preventing } \\
\text { loss of skill }\end{array}$ & $\begin{array}{l}\text { Encouraging } \\
\text { initiative }\end{array}$ \\
\hline speed & 5,7 & & & & & \\
\hline quickness & $1,2,6,7,9,10$ & $3,4,6,1,2,8$ & $3,4,6,8$ & $3,4,6,1,10$ & $3,4,1,6,8,10$ & $3,10,1,4,6,9$ \\
\hline volume/weight & $1,5,6$ & $5,6,1,3,4$ & $3,4,5,6$ & $3,4,5,6$ & & $3,4,5,6$ \\
\hline un-deterioration & $2,5,6$ & $2,5,6$ & & $3,5,10$ & $3,5,10$ & $3,5,10$ \\
\hline few types of operation & $5,9,10$ & $4,5,6,8,9$ & $4,5,6,8,9$ & $4,6,5,9$ & $5,6,8$ & $4,5,6,9,10$ \\
\hline low amount of operation & $5,9,10$ & $3,5,8$ & $3,5,8$ & 3 & $3,5,8$ & $3,5,9,10$ \\
\hline standardization & 5,10 & $3,4,5,6,8$ & $3,4,5,6,8$ & $3,4,6,5$ & $3,4,5,8$ & $3,4,5,6,10$ \\
\hline
\end{tabular}

Table 2

Fuben-eki principles

\begin{tabular}{ll}
\hline 1. Degradation & 7. Fatigue \\
2. Enlargement & 8. Danger \\
3. Increase the number of operations & 9. Disorder \\
4. Increase the amount of operations & 10. Constraint \\
5. Time consumption & 11. Stimulation \\
6. Continuity (Analog) & 12. Less information \\
\hline
\end{tabular}

fuben-eki matrix to support the divergent thinking of the fuben-eki system design. The examples of the benefits shown in the columns of the fuben-eki matrix can also be used to explore benefits.

This paper proposes fuben-eki cards as a support tool for system design. Their layout is designed by referring to idea pop-up cards [21]. A set of fuben-eki cards consists of two types: principle cards and benefit cards (Fig. 5). Principle cards are 12 yellow cards on which the fuben-eki principles are shown; i.e., they present the parameters of inconvenience. Benefit cards are eight green cards that show examples of benefits. A pictogram shown on each card supports users to understand the content intuitively.

\subsection{Support tools for divergent thinking}

For supporting divergent thinking, many systems utilizing computers have been proposed, e.g., Articulation Aid 1 [22], IDEA System [23], Metaphor Machine [24]. Articulation Aid 1 presents spacial arrangement of the words provided by users and encourages users to form new concepts. IDEA System uses a knowledge-base of problem solutions and draws effective analogies with the problem in question. Metaphor Machine, borrowing a leaf of Synectics [25], generates metaphor by referring to a data-base.

Fuben-eki cards are made of paper and do not need a computer. They do not require to use a data-base or a knowledge-base, and do not generate a solution itself or an appropriate hint suited to the problem. Selecting a card and associating the card with new idea are highly dependent on users. However, the cards spend less cognitive resources to use them because the structure and usage of fuben-eki cards are simpler than other methods using a computer.

Although both the fuben-eki matrix and the cards are support tools for fuben-eki system design, they are different in the purpose and the way to use. The fubeneki matrix requires users to model their problem as a contradiction. It systematically provides appropriate principles to solve the problem only if users know the benefit they want to embody. When users want broad ideas and postpone determining benefits to embody, fuben-eki cards will be suitable.

\section{Verifying the utility of the cards without constraints}

This section shows our experiment that evaluated the utility of the cards.

\subsection{Outline}

The examinees consisted of 46 men and women in their teens or twenties without knowledge of fubeneki. They were divided into three groups:

- with PC: with principle cards

- with BC: with benefit cards

- w/o C: without the cards.

The examinees were requested to create ideas to convert the target system into a fuben-eki system using divergent thinking. The above task, called the idea generation task, was conducted twice. The target systems were a microwave oven ( $P$ in Table 3 ) and a wall clock $(Q)$. These systems satisfy the following conditions:

- most people are familiar with and can use them, - the purposes of the systems are clear and limited. 


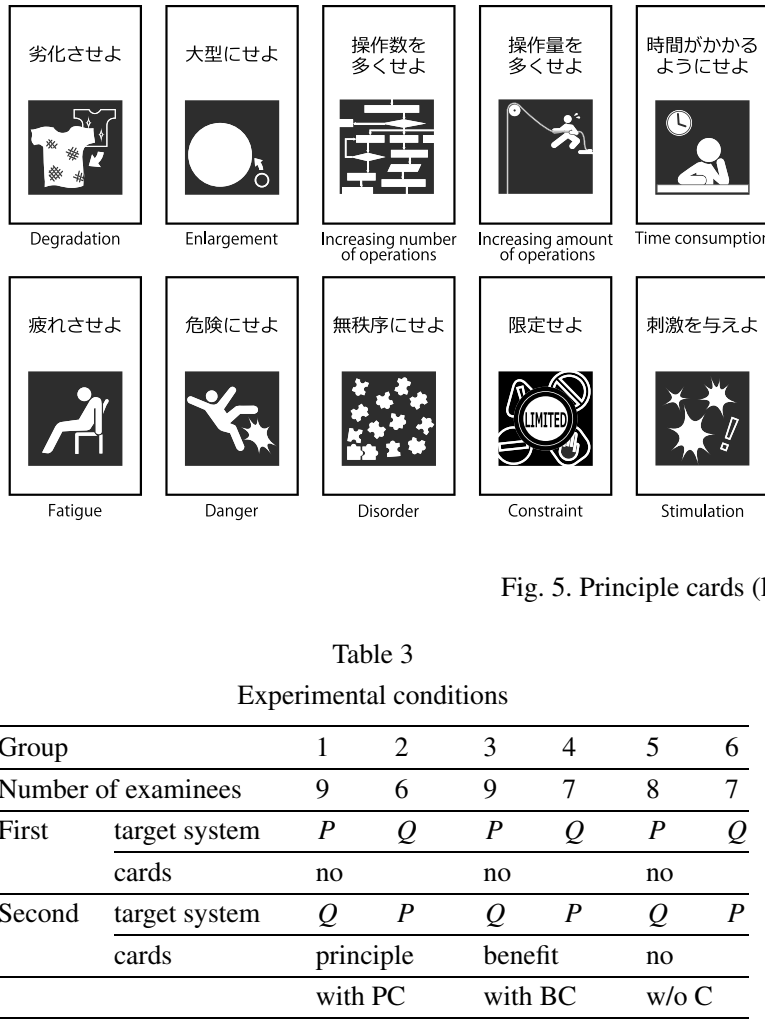

Each group was split into two following the order of the target system (Table 3).

\subsection{Experimental procedure}

i-1 First idea generation task (explanation +20 minutes). The examinees were given a brief outline of fuben-eki and brainstorming. Then each examinee was required to create ideas to reconstruct the target into an inconvenient one and to explore the benefits obtained from the inconvenience by individual brainstorming. They wrote down their ideas and benefits on paper. More than one benefit can be found in each idea.

$i$-2 Self-selection of ideas (2 minutes). The examinees selected their two favorite ideas from their own ideas. These are called selected ideas.

ii-1 Second idea generation task (explanation +20 minutes). The examinees were requested to create ideas to change the other target system into a fuben-eki system in the same way as in $i-1$. Those with PC were permitted to use and to refer to their principle cards. The examinees with $\mathrm{BC}$ were permitted to use and to refer to their benefit cards. The card users were informed that the fuben-eki principles are printed on princi-

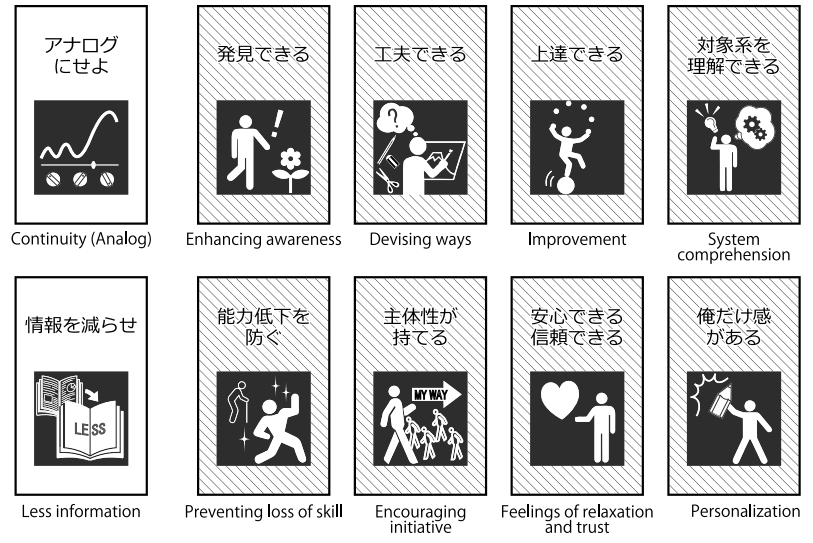

Less information

Encouraging
initiative ple cards and examples of benefits are printed
on benefit cards. They can use the cards in any way.

ii-2 Self-selection of ideas (2 minutes). The examinees selected their two favorite ideas as in $i-2$.

iii Questionnaires. At the end of the experiment, the examinees answered questionnaires about the experiment.

\subsection{Evaluation of ideas and experimental data}

Three persons who had been engaged in study of fuben-eki for more than three years, including the developer of the fuben-eki principles, comprehensively evaluated the selected ideas. They scored each idea in view of both inconvenience and benefits (10 points maximum). Since the degree of feeling inconvenience and benefit varies depending on each person, quantifying the overall degree is not easy. In this paper, for convenience, we assumed the average evaluations of the three experts to be the scores of each idea. Because each examinee selected two ideas in each idea generation task, the average of the two ideas' scores is assumed to be the evaluation score for the examinee. We obtained the following datasets by the above procedure:

- number of ideas created by each examinee in the first and second idea generation tasks (number),

- number of benefits found by each examinee in the first and second idea generation tasks (number),

- evaluation score of each examinee in the first and second idea generation tasks by evaluator (score),

- answers to questionnaires by each examinee (score and free comments). 

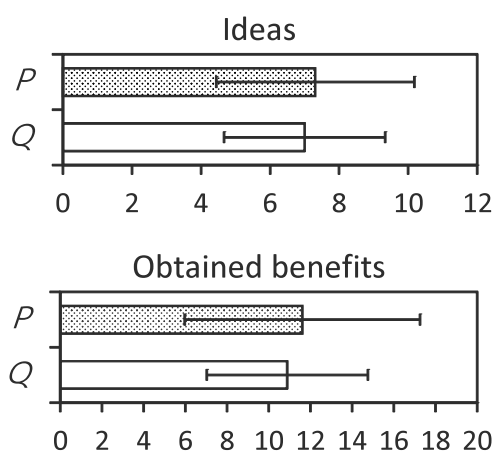

Fig. 6. Number of ideas and obtained benefits.

\subsection{Experimental result 1}

First, examples of ideas are shown below:

- a microwave oven whose heating time can't be set; users must concentrate on the food and find their own preferred heating time,

- a wall clock that needs to be wound every day; users will be fond of it and understand its mechanism.

All examinees were not familiar with the concept of fuben-eki and created ideas without criticism. Thus, some ideas were questionable in view of feasibility and suitability of fuben-eki. In this experiment, for convenience, we counted all ideas and benefits.

Based on the experimental results, we verify the following hypotheses:

$\alpha:$ the cards will increase the number of ideas,

$\beta$ : the cards will increase the number of obtained benefits,

$\gamma$ : the cards will increase the evaluation scores.

Before verifying the hypotheses, we compared the number of ideas and obtained benefits of target system $P$ with those of target system $Q$. Figure 6 shows the number of ideas and obtained benefits of the first idea generation task. An analysis of variance (ANOVA) shows no significant difference between target system $P$ and $Q$, i.e., no difference in the performance of the target systems.

We verified the hypotheses by comparing the difference of the ideas, the obtained benefits, and the evaluation scores between the first and second idea generation tasks. Figure 7 compares them among the three groups. The ANOVA shows no significant difference among the three groups, i.e., no significant difference in the performance even with the cards.

\subsection{Discussion 1}

Hypotheses $\alpha, \beta$, and $\gamma$ are rejected for the following reasons:

- The examinees had to learn how to use the cards, because they were not informed how to use them. Therefore, they couldn't focus on the idea generation task.

- The examinees did not try to interpret the contents of the cards, which were described abstractly. Therefore, they failed to make good use of them.

These reasons suggest that the quantity and the quality of ideas do not change because of a lack of information for an effective way to use the cards.

\section{Verifying the utility of the cards with a constraint of usage}

We experimentally verified hypotheses $\alpha, \beta$, and $\gamma$ introduced in Section 4.4 again by fixing how the cards were used.

\subsection{Outline of experiment 2}

The examinees consisted of 45 men and women in their teens or twenties without knowledge of fubeneki. Table 4 shows the experimental conditions. The w/o C data are the same as those in Section 4.1.

\subsection{Experimental procedure}

The procedure of the experiment was the same as in Section 4.2, except for giving the guidelines as follows:

\section{Guidelines to use principle cards}

- refer to one card to produce an idea,

- explore benefits without cards,

- if no idea is produced, change the card quickly,

- the contents of the cards can be interpreted boldly.

Guidelines to use benefit cards

- produce ideas without cards,

- refer to each card to explore benefits,

- if all the cards have been referred to, move to the next idea,

- the contents of the cards can be interpreted boldly.

Each examinee is encouraged to refer to a card from various viewpoints. 

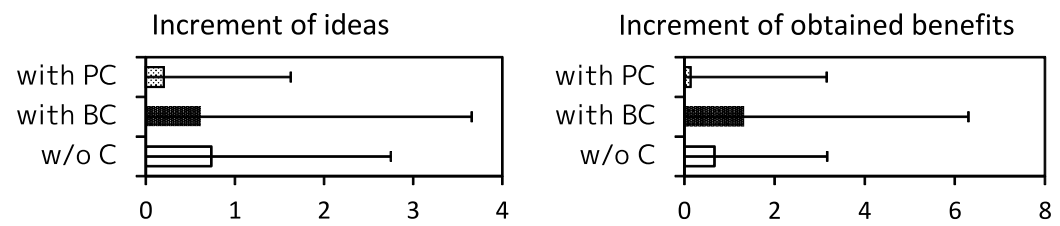

Increment of evaluation scores

Fig. 7. Increment of ideas, obtained benefits, and evaluation scores.
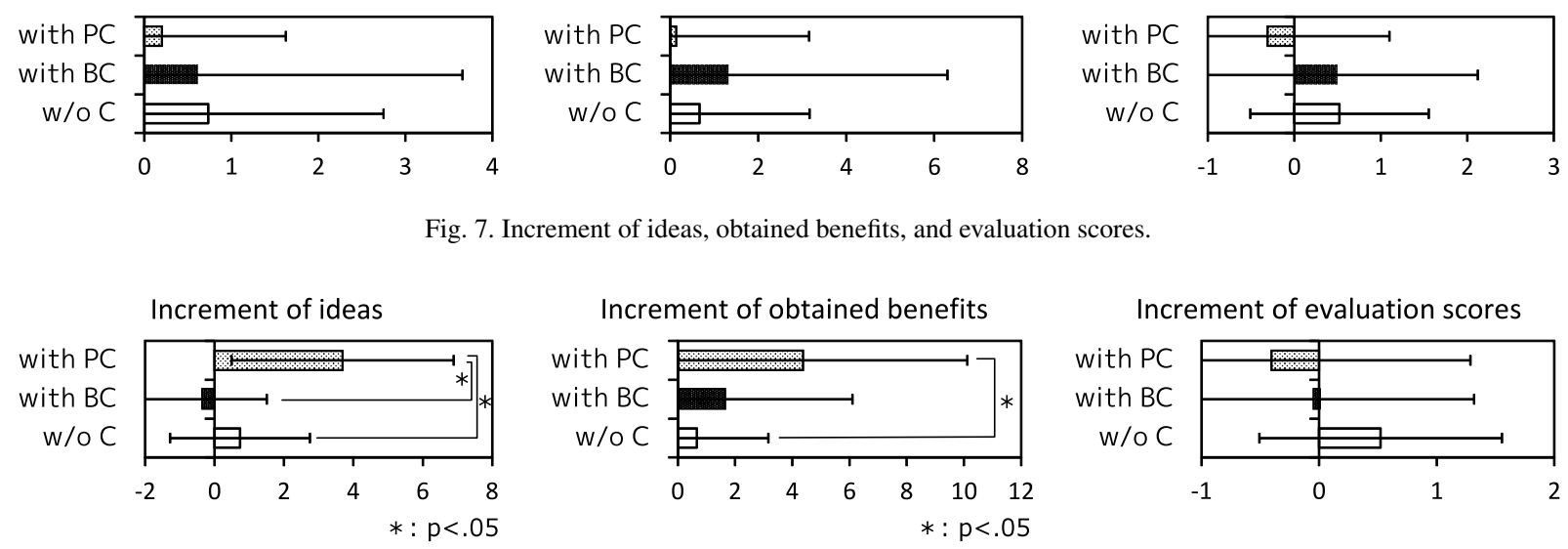

Fig. 8. Increment of ideas, obtained benefits, and evaluation scores with cards used based on guidelines.

Table 4

Experimental conditions

\begin{tabular}{llllllll}
\hline Group & 1 & $2^{\prime}$ & $3^{\prime}$ & $4^{\prime}$ & 5 & 6 \\
\hline Number of examinees & 8 & 8 & 7 & 7 & 8 & 7 \\
\hline \multirow{2}{*}{ First } & target system & $P$ & $Q$ & $P$ & $Q$ & $P$ & $Q$ \\
\cline { 2 - 9 } & cards & no & & no & & no & \\
\hline \multirow{2}{*}{ Second } & target system & $Q$ & $P$ & $Q$ & $P$ & $Q$ & $P$ \\
\cline { 2 - 8 } & cards & principle & benefit & \multicolumn{2}{l}{ no } & \\
\hline & with PC & with BC & \multicolumn{2}{l}{ w/o C } \\
\hline
\end{tabular}

\subsection{Experimental result 2}

Based on our experimental results, we verified hypotheses $\alpha, \beta$, and $\gamma$. Figure 8 shows the increment of the ideas, the obtained benefits, and the evaluation scores between the first and second idea generation tasks. In the results of the increment of ideas, the ANOVA shows that the main effect among groups was significant $(F(2,42)=10.97, p<.001)$. Shaffer's multiple comparison test shows that the value of the PC group is higher than that of the other groups at a $5 \%$ significant level. In the results of the increment of the obtained benefits, the main effect of the different groups was marginally significant $(F(2,42)=2.87$, $p<.10)$. The multiple comparison test shows that the value of the PC group is higher than the w/o C group at a 5\% significant level. The ANOVA shows no significant difference in the results of the evaluation scores.

\subsection{Discussion 2}

Based on the above results, although hypothesis $\gamma$ was rejected, $\alpha$ and $\beta$ are modified as follows: $\alpha^{\prime}$ : Principle cards will increase the number of ideas with the users who follow the guidelines;

$\beta$ ': Principle cards will increase the number of obtained benefits with the users who follow the guidelines.

\subsubsection{The effect of principle cards on quantity}

Principle cards increase the number of ideas and obtained benefits. The following are the possible reasons:

- The fuben-eki principles inspired users to devise fuben-eki systems in various aspects.

- The cards and the guidelines worked as forced association, such as a checklist method [6,26].

- The users were not allowed time to daydream because they had to refer to each card quickly.

In the questionnaires, some examinees who used principle cards commented that they found some inconveniences that they would not have stumbled upon without the cards and enjoyed the idea generation task because it resembled a game. However, others argued that they could only create those ways to alter the target system that were already shown in the cards. These comments suggest that principle cards might limit the thoughts of the users. Another interpretation of the comments is that the cards cover all the ways to alter systems into inconvenient ones.

It remains unclear whether the increase of ideas was caused by both the cards and the guidelines or only the guidelines. In order to make it clear, additional experiments, which compare the other combinations of cards and guidelines, are required. 


\subsubsection{The effect of the guidelines}

Comparing the results described in Section 4.4 and that in 5.3, enforcing users to concentrate on each principle is effective in the amount of ideas. The guidelines were methods to concentrate users on each principle that turned into triggers for creating new ideas.

Of course, those guidelines are not the best or the only method. We will be able to improve the guidelines or investigate other methods. For example, it is not clear how long we should concentrate on each card.

\subsubsection{The effect of the cards on quality}

The evaluation scores were not changed by the cards; although principle cards increased the quantity of ideas, they did not affect the quality of the top ideas.

In this paper, we evaluated only the selected ideas but such evaluation of all ideas as the average scores or the number of good ideas may be beneficial for clarifying the effects of cards.

\subsubsection{The effect of benefit cards}

Benefit cards are, in the first place, not for altering the target systems into inconvenient ones. In the questionnaire, some examinees argued that they could not hit upon ideas by using the cards. In fact, benefit cards did not increase the number of ideas.

We have to note that not only ideas, but also benefits were not increased. The following are the possible reasons. In the context of this paper, as shown in Section 3.1.1, inconvenience and benefits are in a means-ends relation. By means of making target systems inconvenient, they achieve to obtain benefits. It was a hard task to synthesize means (inconvenience) from ends by showing only benefit cards. Unless the means are not obtained, ends are not conceptualized. Consequently, the BC group ended up with the same results as the w/o $\mathrm{C}$ group. The idea generation process shown in Section 3.1.1 did not utilize benefit cards.

\section{Conclusion}

This paper proposed a card-type tool, which borrows a leaf from the fuben-eki principles and the fuben-eki matrix proposed in previous studies, for divergent thinking to embody the benefits of inconvenience. Our fuben-eki cards are classified into two types: principle and benefit. Their utility was verified by analyzing our experimental results. In the experiment, examinees without knowledge of fuben-eki were divided into three groups: with principle cards, with benefit cards, and without cards. All three groups carried out the same tasks.

We obtained the following results by comparing the average values of each group:

- Neither card type affected the performance when how to use them was not fixed.

- Principle cards increased the number of ideas and obtained benefits when they are used based on guidelines that compel users to concentrate on generating ideas from each card.

- Benefit cards had no effect on the performance even if they are used based on the guidelines.

While the cards and the guidelines proposed in this paper can be classified as forced association, the card users can also associate subjective benefits, which vary depending on the person.

Note that only principle cards produce an effect but benefit cards do not. This suggests that the phase to change the target systems into inconvenient ones is the key for idea creation in fuben-eki system design.

Future work will establish a support method to increase the quality of the ideas of fuben-eki systems. This is important for the convergent thinking process. Improving benefit cards and how to use them as well as reconsidering the evaluation method of fuben-eki systems might be effective.

\section{References}

[1] D.A. Norman, Human centered design considered harmful, Interactions 12(4) (2005), 14-19.

[2] H. Kawakami and T. Hiraoka, Incorporation of evolutionary computation for implementing the benefit of inconvenience, International J. of Advancements in Computing Technology 4(22) (2012), 248-256.

[3] H. Kawakami, Inconvenience Utilizing Design, Kagaku-Dojin Publishing Company Inc., 2011, (in Japanese).

[4] J.P. Guilford, The Nature of Human Intelligence, McGrawHill, New York, 1967.

[5] J.P. Guilford, Traits of creativity, in: Creativity and Its Cultivation, H.H. Anderson, ed., Harper, 1959, pp. 61-142.

[6] A.F. Osborn, Applied Imagination: Principles and Procedures of Creative Problem-Solving, Scribner, New York, 1957.

[7] M. Diehl and W. Stroebe, Productivity loss in brainstorming groups: Toward the solution of a riddle, J. of Personality and Social Psychology 53(3) (1987), 497-509.

[8] A.B. VanGundy, Brainwriting for new product ideas: An alternative to brainstorming, J. of Consumer Marketing 1(2) (1983), $67-74$.

[9] H. Kawakami, H. Suto, H. Handa, T. Shiose and O. Katai, A way for designing artifacts based on profit of inconvenience, in: Proc. of 11th Asia-Pacific Workshop on Intelligent and Evolutionary Systems, 2007, CD-ROM. 
[10] E.L. Deci, Intrinsic Motivation, Plenum Publishing Co., Ltd, 1975.

[11] G.J.S. Wilde, Target Risk 2-a New Psychology of Safety and Health-What Works? What Doesn't? And Why..., PDE Publications, 2001.

[12] A. Bandura, Self-Efficacy in Changing Societies, Cambridge University Press, 1995.

[13] K. Naito, H. Kawakami and T. Hiraoka, Design support method for implementing benefit of inconvenience inspired by the theory of inventive problem solving - TRIZ, J. of the Society of Instrument and Control Engineers 49(6) (2013), 595601, (in Japanese).

[14] K. Naito, H. Kawakami and T. Hiraoka, Design support method for implementing benefits of inconvenience inspired by TRIZ, in: Proc. of Conference TRIZ Future 2012, 2012, pp. $351-356$

[15] D.A. Norman, Emotional Design, Basic Books, 2005.

[16] R.A. Finke, T.B. Ward and S.M. Smith, Creative Cognition: Theory, Research, and Applications, MIT Press, 1992.

[17] H. Kitagawa, Implementing a degrading navigation system as an explanatory example of "benefit of inconvenience", in: Proc. of SICE Annual Conference, 2010, pp. 1738-1742.

[18] R. Jugulum and P. Samuel, Design for Lean Six Sigma: A Holistic Approach to Design and Innovation, John Wiley \& Sons, Hoboken, N.J., 2008.
[19] D. Mann, Hands-on Systematic Innovation, CREAX Press, 2002.

[20] K. Naito, H. Kawakami, T. Hiraoka and K. Inui, Support system for embodying benefit of inconvenience by referring to TRIZ, in: Proc. of 2012 Int. Conf. on Humanized Systems, 2012, pp. 175-180.

[21] R. Ishii, "Idea pop-up cards" for idea creation promotion card tool to be able to experience TRIZ easily, in: Proc. of 6th Knowledge Creation Support Symposium, 2009, pp. 186-195, (in Japanese).

[22] K. Hori, A system for aiding creative concept formation, IEEE Trans. on Systems, Man, and Cybernetics 24(6) (1994), 882894.

[23] T. Hayashi and M. Hagiwara, Idea divergent editor using analogy: The IDEA system, International J. of Intelligent Systems 18(11) (2003), 1155-1172.

[24] L.F. Young, The metaphor machine: A database method for creativity support, Decision Support Systems 3(4) (1987), 309317.

[25] W.J.J. Gordon, Synectics: The Development of Creative Capacity, Harper \& Row, 1961.

[26] E.K. Von Fange, Professional Creativity, Prentice-Hall, 1959. 